\title{
Research on automatic current sharing control methods for control power supply
}

\author{
Xian Bin DAl \\ Electrical Engineering Department, Shenyang Institute of Engineering, China
}

\begin{abstract}
High-power switching devices in control power supply have different saturated forward voltage drops and the inconsistency of turning on/off times and they lead to the inconsistency in the external characteristics of the inverter modules in parallel operation. Modules with good performance in external characteristics undertake more currents and lead to overloading status and modules with bad performance in external characteristics stay in light-loading status, which increases the thermal stress of module undertaking more currents and influences the service life of high-power switching devices. Based on the simulation analysis of the small-signal module using control power supply automatic current sharing method, it is able to find out the characteristics of current-sharing loop control, namely, slow response speed of the current-sharing loop, which is beneficial for improving the stability of the entire control power supply system.
\end{abstract}

\section{Preface}

Control power supply mainly provides power supply for secondary system automatic equipment such as smart power substation control, monitoring, protection, signal and adjustment, etc. and it enjoys the reputation as "heart" of the power operation system. If abnormal operation or malfunction occurs to the control power supply, computer monitoring equipment, network communication equipment, intelligent protection and adjustment equipment in the substation will also suffer from abnormal operation or power failure and the power grid will be running in the status free from protection and surveillance. Once accidents happen to the power grid, relay protection will refuse to act or no selective act will be made, which will cause the widespread blackout and major economic losses. Therefore, control power supply is related to the safe and stable operation of the smart substation and the smart power grid and it is the basic equipment for the entire power operation system and its reliability must be guaranteed.

Generally speaking, control power supply is composed of $\mathrm{AC}$ input unit, rectifier module, surveillance unit, DC output and relevant auxiliary units. [1-2] AC input unit is input to supply power for the rectifier module through one loop in the AC switching \& control circuit. Rectifier module is the key device in the power supply system. In general, high-power and high-frequency switching power supply will be used as the rectifier module. As its core circuit -- "inverter bridge" is composed of high-power switching devices. Surveillance unit mainly conducts surveillance and control of the entire operating power supply system and it contains $\mathrm{MCU}$, measurement module, control module, human-machine interactivity module and telecommunication module. Currently, control power supply used in smart substations is being gradually replaced by high-frequency, modularized and intellectualized switching power supply to enable the control power supply to display characteristics such as small volume, high efficiency, small ripple coefficient, rapid dynamic response, high control precision and stack output of module, etc. Nevertheless, some problems also exist such as current sharing/voltage sharing problems among rectifier modules in parallel connection. [3-4]

\section{Equations Current Sharing of Rectifier Module}

Generally speaking, control power supply usually uses high-power, intellectualized and high-frequency switching power supply as its rectifier module and as its core circuit -- "inverter bridge" is composed of highpower switching devices. In order to elevate the output power of the rectifier module, it usually uses multiple inverter bridges in serial connection in operation, which

\footnotetext{
a Corresponding author:dxbcy@126.com
} 
leads to the uneven current/current among devices in parallel connection and it also influences the service life of the high-power switching devices. The high-power switching devices used to form the inverter bridges in parallel connection have different saturated forward voltage drops and the inconsistency of turning on/off times and they lead to the inconsistency in the external characteristics of the inverter modules in parallel operation. Modules with good performance in external characteristics undertake more currents and lead to overloading status and modules with bad performance in external characteristics stay in under-loading status, which increases the thermal stress of module undertaking more currents and influences the service life of highpower switching devices. [5]Therefore, in the design for control power supply, it is necessary to adopt currentsharing technology in order to guarantee the even distribution of power supply stress and thermal stress among the modules to keep one or multiple modules from running in the extreme current status.

General current sharing methods include output impedance method, master-slave control method, automatic current sharing method based on average current value, external current sharing controller method and automatic current sharing method. Though there are many current sharing methods, they often display deficiencies in practical application. [6] Specifically speaking: current sharing precision is too low in output impedance method; it is unable to realize redundancy technique and it fails to well guarantee the reliability of the parallel power supply module system through adopting master-slave control method and automatic current sharing method based on average current value; external current sharing controller method makes system become more complicated and it is not beneficial for converting the technique to practical product. As to automatic current sharing method, it has characteristics such as high current sharing precision, good dynamic response and the possibility of realizing redundancy technique, etc, which makes it become more and more popular. Automatic current sharing method -- in $n$ modules in parallel connection, it takes the module with largest output current as the master module and the others are considered as slave modules. No module is preset manually as the main module and they are sequenced according to the size of current value, namely, module with larger current value automatically becomes the master module. Therefore it is called "automatic current sharing method".

\section{Rectifier Module's Hardware Circuit Design}

Rectifier Module's Hardware Circuit Block Design is as illustrated in Figure 1. It is mainly composed of EMI filter, AC/DC circuit with three-phase passive PFC technique, DC/DC convertor, input/output detection and protection circuit and auxiliary power supply, etc. Based on the aforesaid hardware circuit scheme, the design prototype's parameters are as follows: input -- $\mathrm{AC} 380 \mathrm{~V}$, power factor -- $\cos \varphi \geq 0.98$; output DC voltage range -$0 \sim 250 \mathrm{~V}$, output DC -- 10A.

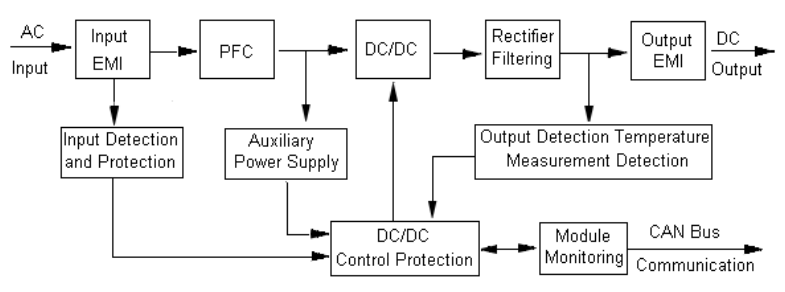

Figure 1. Rectifier Module's Hardware Circuit Block Design

Three-phase Passive PFC Circuit Design -- PFC technique refers to the technique used to restrain harmonic pollution and improve power factor. Passive PFC technique uses passive components to improve power factor and reduce harmonic wave so as to meet with the rectifier power supply module's DC output standard. PFC circuit's overall design principle diagram is as illustrated in Figure 2.

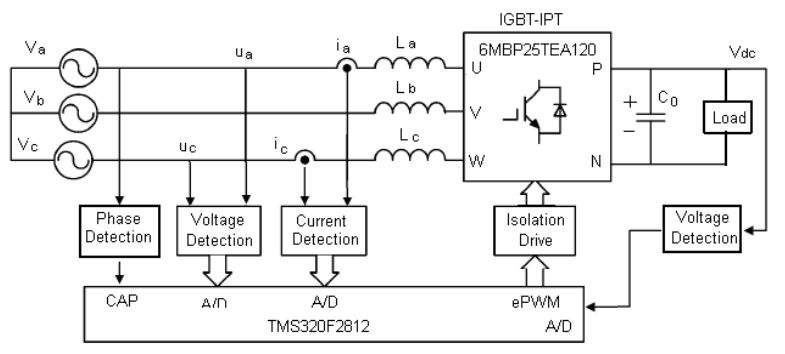

Figure 2. Filter Circuit

PFC Circuit is composed of three-phase rectifier main circuit and digital control circuit. The rectifier main circuit's structure mainly uses Boost type of six-switch \& three-phase PFC circuit and its switch and protection circuit uses IGBT-IPM intelligent module with model number of 6MBP25TEA1200 manufactured by Fuji Company.

The digital control circuit mainly includes electric signal sampling circuit at DC/AC sides, sampling conditioning circuit, control processor (TMS320F2812 fixed-point 32-bit DSP chip manufactured by TI Company) and IMP drive and isolation .Sampling quantity at $\mathrm{DC} / \mathrm{AC}$ sides mainly includes phase-a/phase-b current and voltage at AC side, phase position of phase-a voltage and output voltage at DC side. Sampling quality is finished by Hall current, voltage transformer and voltage sensor manufactured by LEM Company.

\section{Modeling in Automatic Current Sharing Method}

Current-sharing controller's circuit diagram in automatic current sharing method is as illustrated as Figure 3. In the 
Figure, U0 is follower for output voltage detection and it isolates load from influencing the voltage detection; $\mathrm{U} 1$ is current sharing loop amplifier; U2 is voltage loop amplifier; U3 is current loop amplifier.

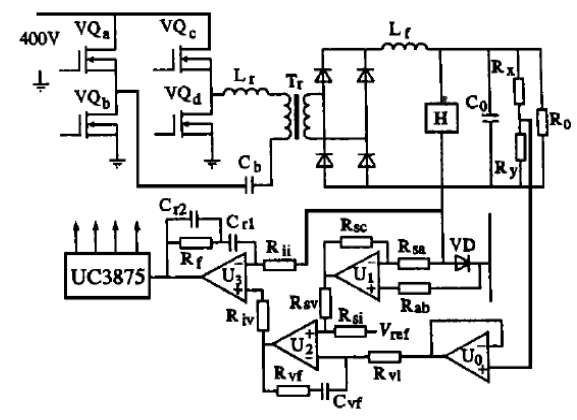

Figure 3. Current Sharing Controller's Circuit Diagram

Control block diagram based on small-signal automatic current sharing method is as illustrated as Figure 4.

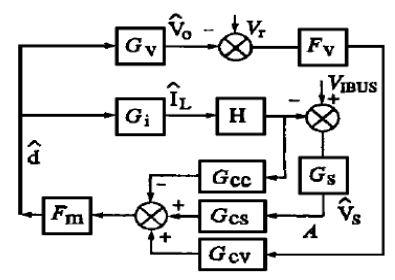

Figure4. Control Block Diagram of Small-signal Automatic Current Sharing method

$$
\begin{gathered}
T_{S}=F_{m} G_{c s} G_{s} H G_{i} \\
\left.G_{c s}=1+W_{t} \frac{S+W_{z}}{S\left(S+W_{p}\right)}\right) \\
G_{s}=40 \frac{R_{v i}}{R_{v s}+R_{v i}}\left(1+\frac{S R_{v f} C_{v f}+1}{S R_{v 1} C_{v f}}\right) \\
G_{i}=\frac{N V_{i n}}{Z_{f}+R_{d}}
\end{gathered}
$$

In formula (1) to (4), $\mathrm{H}$ is the transfer function from detection current to voltage signal, Fm is pulse width modulation transfer function, $\mathrm{Gv}$ is transfer function of output voltage, Gi is transfer function of inductive current, Gs is transfer function of current sharing amplifier, Gcc is current loop's current amplifier transfer function, Gcs is current sharing loop's current amplifier transfer function and Gcv is voltage loop's current amplifier transfer function.

\section{Simulation and Analysis of Automatic Current Sharing Method}

In this simulation, it uses six modules in parallel connection for experiment. In circuit in Figure 3, parameters of all devices are as follows: input - $380 \mathrm{~V}$, output- $220 \mathrm{~V}$, switching frequency fs $-50 \mathrm{kHz}$, equivalent ratio of main transformer $\mathrm{N}-10 / 20$, primary resonant inductor $\mathrm{Lr}-20 \mu \mathrm{H}$, output filtering inductor $\mathrm{Lf}-160 \mu \mathrm{H}$, output filtering capacity Co $-1200 \mu \mathrm{H}$, current measurement conversion ratio - 0.012 (V/A), current sampling gain $\mathrm{H}$ - 20, Fm - 0.20;

Simulation is conducted through MATLAB and bold plots obtained for the current sharing loop's open-loop gain are as illustrated as Figure 5 and 6. Based on the analysis of bold plots of current sharing loop's transfer function, crossover frequency basically occurs at $500 \mathrm{kHz}(\mathrm{rad} / \mathrm{s})$ with slow dynamic response speed.

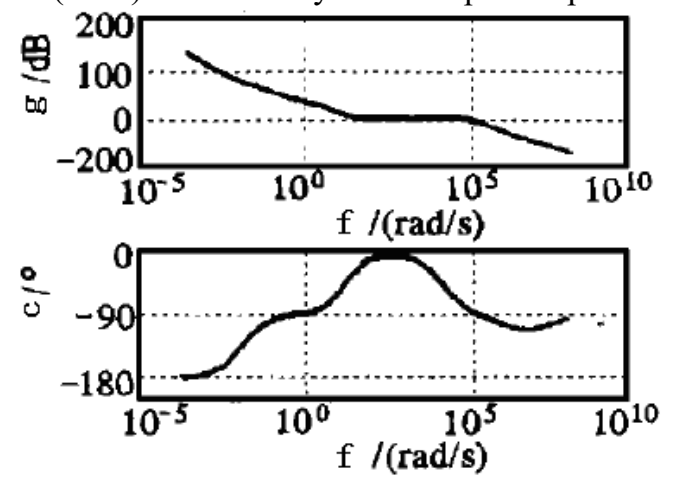

Figure 5. Bolt Plots in TS Full Loading

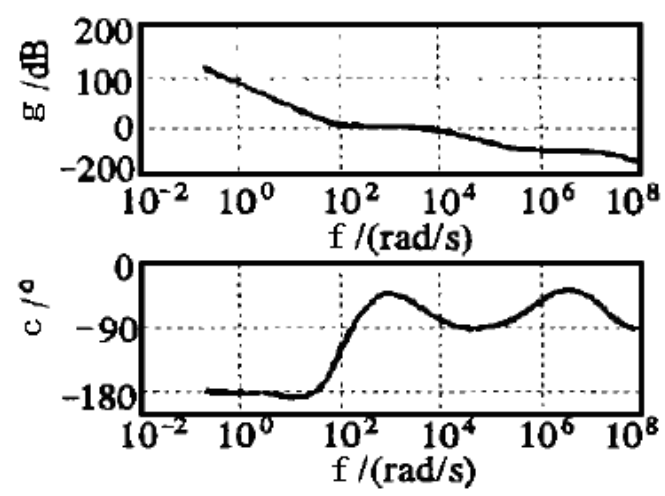

Figure 6. Bolt Plots in TS Light Loading

\section{Conclusion}

Based on analysis of small-single module in automatic current sharing method, it is able to find out current sharing loop control and slow response speed, which is beneficial for controlling the stability of the entire control power supply. The continuous development of electric and electronic techniques will enable current sharing techniques to become more and more mature and perfect and it will inevitably promote control power supply to develop towards larger output power.

\section{Acknowledgements}

The paper is financially aided by "General Scientific Research Project (L2014515) among Colleges and Universities in Liaoning Province" Fund. 


\section{References}

1. Zijing Yang, Qiwei Lu, Duolun Wang, Cong Wang, DSP-based Digital Control Electric Operation Power Supply Development, Power Electronics, 2012(7): 73-75.

2. HIRAKI E, TANAKA T, NAKAOKA M. Zerovoltageand zero-current soft-switching PWM Inverter.EPE 2012 Dresden, ISBN:90-7 58115-08-5.

3. Wei Wei, Jun Li, Lanjie Wei, Research on A Soft Switching Electric Operation Power Supply, Power Electronics, 2013(11): 47-49.

4. T.-F. Wu, H.-M. Hsieh, Y.-E. Wu, et al, Parallelinverter system with failure isolation and hot-swap features, IEEE Trans. Industry Applications, 2007, 43 (5): $1329 \sim 1340$.

5. Chen LiangLiang,XiaoLan, Yan Yanguang. DC LooP-Current detecting and Restraining Methods For Parallel Inverters System. Trans.of NJUA\&A, 2004, 21(1):1-6.

6. Bo Zhang, Parallel Connection and Current Sharing Technique for High-frequency Switching Power Supply in Power Operating System, The World of Power Supply, 2012(3): 26-29. 